\title{
Effects of the Temperament and Character on Depression, Anxiety, and Maternal-Fetal Attachment in High-Risk Pregnant Women
}

\author{
Sang Hyup Jung, MD1, Jin Gon Bae, MD2, Sung Won Jung, MD, PhD1, \\ Min Kyung Kim, MA1, Hee Cheol Kim, MD, PhD1 and Jung Bum Kim, MD, PhD1 \\ 1) Department of Psychiatry, Keimyung University School of Medicine, Daegu, Korea \\ 2) Department of Obstetrics and Gynecology ${ }_{2}$, Keimyung University School of Medicine, Daegu, Korea
}

Objective : High-risk pregnant women are exposed to the physical and emotional crisis associate with the condition of high-risk pregnancy, making these women emotionally unstable, exhibiting such conditions as depression and anxiety. The aim of this study was to examine what effects the temperament and character of high-risk pregnant women had on their depression, anxiety, and maternal-fetal attachment.

Methods : A hundred and five high-risk pregnant women were recruited in the maternity ward of the Keimyung University medical center. All subjects were administered a sociodemographic and obstetric questionnaire, Temperament and Character Inventory, Beck Depression Inventory-II, State Trait Anxiety Inventory-State, and Maternal-Fetal Attachment Scale.
Results : First, higher scores on the harm-avoidance scale and lower scores on the self-directedness scale were associated with higher depression scores. Second, higher scores on the harm-avoidance and reward dependence scale were associated with higher anxiety scores. Lastly, higher scores on the cooperativeness scale were associated with higher maternal-fetal attachment scores.

Conclusion : Temperament and character in high-risk pregnant women were associated with the degree of depression, anxiety, maternal-fetal attachment. Based on these findings, it is suggested that examining temperament and character in advance and screening for vulnerability in pregnant women can help to prevent emotional problems for high-risk pregnant women.
Table 1. Sociodemographic characteristics

\begin{tabular}{|l|c|}
\hline \multicolumn{2}{|c|}{ Subjects(N=105) } \\
\hline Age, year & $32.60 \pm 3.95$ \\
\hline Marital status & \\
Unmarried & $1(0.95 \%)$ \\
Married & $104(99.05 \%)$ \\
\hline Marital period & \\
$<1$ year & $13(12.6 \%)$ \\
$1-2$ years & $21(20.4 \%)$ \\
$2-4$ years & $37(35.9 \%)$ \\
$>4$ years & $32(31.1 \%)$ \\
\hline Living arrangement & \\
Spouse & $59(56.2 \%)$ \\
Spouse, children & $41(39.0 \%)$ \\
Spouses, children, roommate & $5(4.8 \%)$ \\
\hline Educational level & \\
Middle school & $1(1.0 \%)$ \\
High school & $22(21.0 \%)$ \\
University and over & $82(78.1 \%)$ \\
\hline Household income (for 1month, $甘 10,000)$ & \\
$\leq 100$ & $7(6.9 \%)$ \\
$101-200$ & $13(12.4 \%)$ \\
$201-300$ & $24(22.9 \%)$ \\
$301-400$ & $25(23.8 \%)$ \\
$401-500$ & $9(8.6 \%)$ \\
$501 \leq$ & $23(21.9 \%)$ \\
\hline Current number of children & $0.50 \pm 0.67$ \\
\hline
\end{tabular}

Table 2. Obstetric characteristics

\begin{tabular}{|l|c|}
\hline \multicolumn{2}{|c|}{ Subjects (N=105) } \\
\hline Gestational period, week & $29.35 \pm 5.85$ \\
\hline Planned pregnancy & $93(90.3 \%)$ \\
Yes & $10(9.7 \%)$ \\
No & \\
\hline Current obstetric diagnosis & $68(64.8 \%)$ \\
Preterm labor & $6(5.7 \%)$ \\
Premature rupture of membrane & $31(29.5 \%)$ \\
Cervical incompetence & $7(6.7 \%)$ \\
Placenta previa & $2(1.9 \%)$ \\
Preeclampsia & $8(7.6 \%)$ \\
Complications of pregnant women & \\
(gestational diabetes, hypertension, & \\
tuberculosis, etc) & $3(2.9 \%)$ \\
\hline Fetal growth retardation & $8(7.6 \%)$ \\
\hline Other &
\end{tabular}

Table 3. TCI, BDI-II, STAI-S, MEAS scores

\begin{tabular}{|l|l|}
\hline \multicolumn{1}{|c|}{ Mean \pm SD } \\
\hline TCI & $30.32 \pm 8.87$ \\
Hovelty seeking & $37.70 \pm 10.03$ \\
Reward dependence & $45.55 \pm 8.07$ \\
Persistence & $39.78 \pm 10.46$ \\
Self-directedness & $48.13 \pm 10.19$ \\
Cooperativeness & $54.05 \pm 10.01$ \\
Self-transcendence & $21.61 \pm 10.45$ \\
\hline BDI-II & $12.86 \pm 6.90$ \\
\hline STAI-S & $50.50 \pm 11.67$ \\
\hline MEAS & $74.01 \pm 10.99$ \\
\hline TCI : Temperament and Character Inventory, \\
BDI-II : Beck Depression Inventory-II, STAI-S: \\
State Trait Anxiety Inventory-state, MFAS : \\
Maternal-fetal attachment scale
\end{tabular}

Table 4. Multiple regression analysis of TCI for BDI-II, STAI-S, MFAS

\begin{tabular}{|l|c|c|c|c|c|c|}
\hline & \multicolumn{2}{|c|}{ BDI-II $\left(R^{2}=0.402\right)$} & \multicolumn{2}{c|}{ STAI-S $\left(R^{2}=0.200\right)$} & \multicolumn{2}{c|}{ MAFS $\left(R^{2}=0.186\right)$} \\
\cline { 2 - 7 } & Estimated $\beta$ & $p$ & Estimated $\beta$ & $p$ & Estimated $\beta$ & $p$ \\
\hline TCI & & & & & & \\
Novelty seeking & -0.036 & 0.723 & -0.042 & 0.715 & -0.184 & 0.119 \\
Harm avoidance & 0.379 & $0.002+$ & 0.317 & $0.018^{*}$ & 0.022 & 0.872 \\
Reward dependence & 0.072 & 0.449 & 0.262 & $0.018^{*}$ & 0.097 & 0.382 \\
Persistence & 0.144 & 0.201 & 0.047 & 0.716 & 0.178 & 0.172 \\
Self-directedness & -0.351 & $0.005 \dagger$ & -0.158 & 0.260 & -0.053 & 0.707 \\
Cooperativeness & -0.038 & 0.716 & -0.117 & 0.330 & 0.276 & 0.024 * \\
Self-transcendence & 0.127 & 0.193 & -0.203 & 0.071 & 0.158 & 0.161 \\
\hline
\end{tabular}

* : $\mathrm{p}<0.05$ by Multiple regression analysis, $\uparrow: \mathrm{p}<0.01$ by Multiple regression analysis. $\mathrm{TCl}$ : Temperament and Character Inventory, BDI-II : Beck Depression Inventory-II, STAI-S : State Trait Anxiety Inventory-state, MFAS : Maternal-fetal attachment scale 